\title{
Bromeliaceae da Ilha Grande, RJ: revisão da lista de espécies
}

\author{
André Felippe Nunes-Freitas ${ }^{1,3}$, Thereza Christina da Rocha-Pessôa ${ }^{2}$, \\ Aline dos Santos Dias ${ }^{1}$, Cristina Valente Ariani ${ }^{2}$ \& Carlos Frederico Duarte da Rocha ${ }^{2}$ \\ ${ }^{1}$ Departamento de Ciências Ambientais, Instituto de Florestas, \\ Universidade Federal Rural do Rio de Janeiro - UFRRJ, \\ Rodovia BR-465 Km 7, CEP 23890-000, Seropédica, RJ, Brasil \\ ${ }^{2}$ Laboratório de Ecologia de Vertebrados, Departamento de Ecologia, \\ Instituto de Biologia Roberto Alcântara Gomes, Universidade do Estado do Rio de Janeiro - UERJ, \\ Rua São Francisco Xavier, 524, CEP 20550-011, Rio de Janeiro, RJ, Brasil \\ ${ }^{3}$ Autor para correspondência: André Felippe Nunes-Freitas,e-mail: afnfreitas@ufrrj.br
}

NUNES-FREITAS, A.F., ROCHA-PESSÔA, T.C., DIAS, A.S., ARIANI, C.V. \& ROCHA, C.F.D. Bromeliaceae of Ilha Grande: species checklist review. Biota Neotrop. 9(2): http://www.biotaneotropica.org.br/v9n2/en/abs tract?inventory+bn01209022009.

\begin{abstract}
Ilha Grande, located at the Southern coast of Rio de Janeiro State, is constantly punctuated as an important area for conservational purposes, specially because it is a representative fragment of Atlantic forest and presents a high number of studies about the local fauna and flora. The aim of this study is to analyze the current knowledge of Bromeliaceae family at this island, and understand how the increase of the family's species list occurred through out the amount of time and sampling effort of this study. A total of 58 species of Bromeliaceae were listed for Ilha Grande, and 47 of them were sampled using only the methodology adopted at this study and, therefore, constitute new records for Ilha Grande.
\end{abstract}

Keywords: Atlantic forest, sampling effort, species-area curve, sampling methods.

NUNES-FREITAS, A.F., ROCHA-PESSÔA, T.C., DIAS, A.S., ARIANI, C.V. \& ROCHA, C.F.D. Bromeliaceae da Ilha Grande: revisão da lista de espécies. Biota Neotrop. 9(2): http://www.biotaneotropica.org.br/v9n2/pt/ abstract?inventory+bn01209022009.

Resumo: A Ilha Grande, localizada no sul do Estado do Rio de Janeiro, vem se destacando como importante área para fins conservacionistas, especialmente por ser um fragmento representativo de Floresta Atlântica e por apresentar um elevado número de estudos sobre a flora e a fauna local. Este estudo visa analisar o atual status do conhecimento sobre a família Bromeliaceae nesta localidade e analisar o incremento da lista de espécies desta família ao longo do tempo de estudo e do esforço amostral. Foram registradas 58 espécies de Bromeliaceae para a Ilha Grande, das quais 47 foram amostradas apenas com a metodologia empregada neste estudo e constituem registros novos para a Ilha Grande.

Palavras-chave: Floresta Atlântica, esforço amostral, curva espécies-área, métodos de amostragem. 


\section{Introdução}

Nos últimos anos, em virtude do investimento em levantamentos florísticos e consequente aumento do conhecimento da composição florística de regiões anteriormente pouco estudadas (e.g., Fontoura et al. 1991) houve um aumento de publicações sobre flórulas e descobertas de novas espécies. No entanto, a maior parte dos remanescentes florestais e das Unidades de Conservação ainda não apresenta sequer uma lista preliminar de espécies locais (Prance et al. 2000).

A família Bromeliaceae, com distribuição tipicamente neotropical (com exceção de uma espécie que ocorre no oeste da África) (Porembski \& Barthlott 1999), pode ser considerada uma das famílias mais representativas da flora brasileira, especialmente da Floresta Atlântica (Martinelli 2006). Esta formação concentra um grande número de espécies (Smith 1955), guardando uma considerável parcela da riqueza de espécies de Bromeliaceae, inclusive com um elevado grau de endemismo, especialmente no Rio de Janeiro (Fontoura et al. 1991, Martinelli 2006), apesar da reduzida cobertura e da elevada degradação da Floresta Atlântica neste Estado (Rocha et al. 2003).

A Ilha Grande, localizada no litoral sul do estado do Rio de Janeiro, é um dos mais importantes remanescentes de Floresta Atlântica do sudeste brasileiro, apresentando cerca de 50\% dos seus 19.000 ha cobertos por esta formação (Alho et al. 2002). Além de florestas primárias, uma enorme variedade de habitats, que incluem florestas secundárias em diferentes estágios de regeneração, restingas, mangues, brejos e florestas alagadas, são encontrados na Ilha Grande (Maciel et al. 1984; Araújo \& Oliveira 1988), fazendo com que a área seja descrita como um verdadeiro mosaico vegetacional. Essa elevada heterogeneidade fisionômica permite que a ilha possua uma alta diversidade de diferentes grupos, tais como Bromeliaceae (Rocha et al. 2003), elevando assim a sua importância para a conservação.

No presente estudo, avaliamos o status sobre o conhecimento da ocorrência de espécies de bromélias na Ilha Grande, visando responder às seguintes questões: i) Qual a composição de espécies de Bromeliaceae para a Ilha Grande? ii) Como tem sido o incremento no número de espécies conhecidas para a área, em função do tempo? e iii) Qual a curva espécie-área para a Ilha, baseada no acúmulo de parcelas de amostragem?

\section{Material e Métodos}

\section{1. Área de estudo}

O estudo foi realizado na Ilha Grande $\left(23^{\circ} 10^{\prime} 33,27^{\prime \prime} \mathrm{S}\right.$ e $44^{\circ} 22^{\prime} 38,37^{\prime \prime}$ O e $23^{\circ} 10^{\prime} 09,12^{\prime \prime}$ S e $44^{\circ} 05^{\prime} 15,84^{\prime \prime}$ O), localizada no município de Angra dos Reis, no Rio de Janeiro. A região apresenta cerca de 19.000 ha com relevo bastante acidentado, sendo seus dois pontos mais altos o Pico do Papagaio (959 m) e a Serra do Retiro (1.031 m) (Maciel et al., 1984). O clima é quente e úmido, com temperatura média de $22,5^{\circ} \mathrm{C}$, sendo a máxima média $25,7^{\circ} \mathrm{C}$, registrada para o mês de fevereiro e a mínima média $19,6^{\circ} \mathrm{C}$, com registro para o mês de julho (Bittencourt \& Rocha 2002). A pluviosidade anual média da região é de aproximadamente $2.200 \mathrm{~mm}$ (Rocha-Pessôa $\&$ Rocha 2008).

$\mathrm{Na}$ Ilha são encontradas diferentes formações vegetais, tais como restingas, costões rochosos, manguezais, matas alagadas e de encosta, contribuindo para que a flora varie de acordo com as diferentes formações presentes (Araújo \& Oliveira 1988). A cobertura vegetal predominante pode ser classificada como Floresta Ombrófila Densa (Veloso et al. 1991), ocupando 46,7\% da vegetação, sendo formada pela floresta original, em geral, em localidades acima dos $400 \mathrm{~m}$ de altitude (Alho et al. 2002). No entanto, mais da metade da cobertura vegetal da Ilha Grande encontra-se em diferentes níveis de regeneração após o término de diferentes ciclos econômicos (Araújo \& Oliveira 1988). As formações secundárias encontram-se, em geral, em estado avançado de regeneração e cobrem a maior parte de suas encostas. Nessas localidades são encontradas comumente espécies pioneiras, como do gênero Miconia (Melastomataceae) (Oliveira 1999) ou extensas áreas cobertas por samambaial (Gleichenia sp) ou campinzais (em geral Melinis minutiflora Beauv., Hypolytrum schraderianum Nees e Panicum maximum Jacq.).

\section{Metodologia}

A amostragem deste estudo foi realizada com o auxílio do programa GPS Track Maker® (Ferreira Jr 2004), com a demarcação de uma grade hipotética composta de linhas longitudinais e latitudinais, distando uma da outra um minuto (aproximadamente 1,7 km). Foram selecionados 39 pontos de interseção (doravante unidades amostrais - UA) entre as linhas longitudinais e latitudinais, localizados tanto no lado oceânico quanto no lado continental da ilha. Em cada UA, no período compreendido entre os anos de outubro de 2000 e agosto de 2005 , foram estabelecidas 30 parcelas de amostragem de $100 \mathrm{~m}^{2}$ $(10 \times 10 \mathrm{~m})$, feitas em linha e distantes entre si em $10 \mathrm{~m}$, aproximadamente na mesma altitude, buscando acompanhar a curva de nível local. Em cada parcela foram registradas as espécies de bromeliáceas presentes e estimadas a abundância total de cada espécie através da contagem do número de rosetas. As espécies epifíticas foram contabilizadas com o auxílio de binóculos ou através de acesso direto ao dossel, quando possível.

Para elaborar a lista de espécies, foram utilizados os dados obtidos a partir de três fontes distintas: 1) metodologia empregada neste estudo; 2) base de dados do Projeto Ecologia de Comunidades de Bromeliaceae da Ilha Grande, que vem sendo realizado nesta localidade desde julho de 1995 (Laboratório de Ecologia de Vertebrados do Departamento de Ecologia da UERJ) (Rocha et al., 2000); e 3) listagens florísticas publicadas para a área: Araújo \& Oliveira (1988), Fontoura et al. (1991), Almeida et al. (1998), Rocha et al. (2000) e Matinelli et al. (2008).

Para a confirmação da identidade dos taxa, foram efetuadas visitas aos principais herbários do Estado do Rio de Janeiro (Herbário do Museu Nacional [R], Herbário Alberto Castellanos/ FEEMA [GUA], Herbarium Bradeanum [HB] e Herbário da Fundação Jardim Botânico do Rio de Janeiro [RB]). O material testemunho das espécies coletadas foi depositado no Herbário do Museu Nacional (R) para posterior identificação.

Para compreender o incremento na riqueza de espécies de Bromeliaceae da Ilha Grande ao longo do tempo, os dados levantados no presente estudo foram comparados aos dos trabalhos publicados desde Araújo \& Oliveira (1988). Nesta análise, foram excluídas as espécies não identificadas ao nível específico, com exceção daquelas que estão em fase de descrição e de publicação. Para análise do aumento da riqueza de espécies com o esforço amostral realizado neste estudo, foi utilizado o método da curva do coletor (Magurran 1988), fazendo o ajuste de curva de forma a saber a função matemática (linear, de potência e logarítmica) que melhor explicasse a relação entre o esforço amostral e a riqueza, e considerando a que exibisse o maior coeficiente de determinação $\left(\mathrm{R}^{2}\right)$ como a de melhor ajuste.

A ocorrência das espécies nos Estados da União foi determinada através de consulta à bibliografia corrente (Fontoura et al. 1991; Martinelli et al. 2008) e as bases de dados disponíveis no SpeciesLink do CRIA (www.cria.org.br). Para categorizarmos as espécies nas diferentes categorias de ameaça, foram utilizadas as informações existentes nas listas oficiais de espécies ameaçadas de extinção estaduais (Rio Grande do Sul - SEMA, 2002; Santa Catarina - Klein, 1990; Paraná - SEMA, 1995; São Paulo - SEMA, 2004; Espírito 
Santo - IEMA, 2005) e federal (IBAMA, 1992) publicadas. Quando as categorias de duas ou mais listas eram conflitantes, optou-se por utilizar a que correspondia a categoria com maior grau de ameaça.

\section{Resultados}

Como resultado dos estudos realizados na região, foram registradas 58 espécies de Bromeliaceae, distribuídas em 16 gêneros, pertencentes às três subfamílias (Pitcairnioideae, Tillandsioideae e Bromelioideae) (Tabela 1). Dentre estas, Tillandsioideae apresentou maior riqueza de espécies ( $\mathrm{S}=29 ; 50,0 \%$ das espécies) em seguida Bromelioideae ( $\mathrm{S}=28 ; 48,3 \%$ ), enquanto a subfamília Pitcairnioideae foi representada por apenas uma espécie $(1,7 \%$ ) (Tabela 1). Das 58 espécies, uma do gênero Tillandsia constitui um taxon ainda inédito para a ciência. $\mathrm{O}$ gênero que apresentou maior número de espécies foi Vriesea (18 spp.), seguido de Tillandsia (11 spp.) e Aechmea (7 spp.) (Tabela 1). A comunidade de bromélias da Ilha Grande representa 19,2\% das 302 espécies registradas para o Estado do Rio de Janeiro e a 7,2\% das 803 espécies listadas para a Floresta Atlântica (veja Martinelli et al. 2008). Das 58 espécies registradas, três não foram ainda identificadas por não terem sido amostradas em período reprodutivo (Tillandsia sp. 1 e sp. 2 e uma espécie de Bromeliaceae indeterminada; Tabela 1).
A primeira relação de espécies de Bromeliaceae para a Ilha Grande se restringiu a área da Reserva Biológica Estadual da Praia do Sul (RBEPS) (Araújo \& Oliveira 1988), na qual foram relacionadas 19 espécies. Posteriormente, Fontoura et al. (1991), no levantamento da família no Estado do Rio de Janeiro, listaram para a Ilha Grande 16 espécies, havendo o acréscimo de duas espécies não registradas por Araújo \& Oliveira (1988). Almeida et al. (1998), no levantamento realizado para região da Vila Dois Rios, registraram 19 espécies, das quais 12 consistiam em registros novos para a Ilha. Rocha et al. (2000), referiram 46 espécies para a Ilha Grande, baseando sua lista em coletas de campo e de herbários e a levantamento bibliográfico, onde foram incluídos oito novos registros de espécies. Com a metodologia empregada neste estudo, foi registrado um total de 47 espécies, sendo que $12(20,7 \%)$ são registros novos para a localidade. A Figura 1 apresenta o incremento da riqueza de espécies em relação ao tempo de estudos na Ilha Grande.

Houve um incremento relativamente lento de espécies com o aumento do tamanho amostral $(\mathrm{N}=1.170$ parcelas em 39 unidades amostrais). O valor da riqueza de espécies amostradas ( $\mathrm{S}=47 ; 81,0 \%$ do total) chegou próximo ao número total de espécies listadas para a ilha $(\mathrm{N}=58)$ (Figura 2). A curva de ajuste que melhor explicou esse incremento foi a de função logarítmica, com um coeficiente de determinação ajustado de $\mathrm{R}^{2}=92,99 \%$ (Figura 2).

Tabela 1. Lista de espécies de Bromeliaceae registradas para a Ilha Grande, Angra dos Reis, RJ, até o presente momento, categoria de ameaça (CA) e as respectivas fontes do registro para cada uma das espécies.

Table 1. List of Bromeliaceae species registered for Ilha Grande, Angra dos Reis, RJ, until the present moment, threath category and its respective font of register for each bromeliad species.

\begin{tabular}{|c|c|c|c|c|c|c|c|}
\hline Espécies & $\mathbf{A}$ & B & $\mathbf{C}$ & D & $\mathbf{E}$ & $\mathbf{C A}$ & Ocorrência no Brasil \\
\hline \multicolumn{8}{|l|}{ Subfamília Pitcairnioideae } \\
\hline Pitcairnia flammea Lindl. & $\mathrm{x}$ & $\mathrm{x}$ & $\mathrm{x}$ & $\mathrm{x}$ & $\mathrm{x}$ & NA & BA, MG, ES, RJ, SP, PR, SC \\
\hline \multicolumn{8}{|l|}{ Subfamília Tillandsioideae } \\
\hline Tillandsia sp. $1 * *$ & & & & & $\mathrm{x}$ & - & - \\
\hline Tillandsia sp. $2 * * *$ & & & & & $\mathrm{x}$ & - & - \\
\hline Tillandsia dura Baker & & & & $\mathrm{x}$ & $\mathrm{x}$ & NA & ES, RJ, SP, SC \\
\hline Tillandsia gardneri Lindl. & $\mathrm{x}$ & $\mathrm{x}$ & $\mathrm{x}$ & $\mathrm{x}$ & $\mathrm{x}$ & VU & PI, CE, PB, PE, AL, SE, BA, MG, ES, RJ, SP, PR, SC, RS \\
\hline Tillandsia geminiflora Brongn. & $\mathrm{x}$ & $\mathrm{x}$ & $\mathrm{x}$ & $\mathrm{x}$ & $\mathrm{x}$ & VU & PB, PE, BA, DF, GO, MG, ES, RJ, SP, PR, SC, RS \\
\hline Tillandsia mallemontii Glaziou ex Mez & & & & & $\mathrm{x}$ & $\mathrm{EP}$ & RN, BA, RJ, SP, PR, SC, RS \\
\hline Tillandsia recurvata $(\mathrm{L}.) \mathrm{L}$. & & & & & $\mathrm{x}$ & NA & CE, RN, PB, PE, AL, SE, BA, MG, ES, RJ, SP, PR, SC, RS \\
\hline Tillandsia stricta Sol. & & & $\mathrm{x}$ & $\mathrm{x}$ & $\mathrm{x}$ & NA & $\begin{array}{l}\text { CE, PB, PE, AL, SE, BA, MG, ES, RJ, SP, PR, SC, MS, MT, } \\
\text { GO, RS }\end{array}$ \\
\hline Tillandsia tenuifolia $\mathrm{L}$. & $\mathrm{x}$ & & & $\mathrm{x}$ & $\mathrm{x}$ & VU & $\begin{array}{l}\text { MS, MT, GO, RO, PA, CE, PB, PE, AL, SE, BA, MG, ES, } \\
\text { RJ, SP, PR, SC, RS }\end{array}$ \\
\hline Tillandsia tricholepis Baker & & & & & $\mathrm{x}$ & $\mathrm{EP}$ & CE, PE, MG, RJ, SP, PR, RS \\
\hline Tillandsia usneoides (L.) L. & & & $\mathrm{x}$ & $\mathrm{x}$ & $\mathrm{x}$ & VU & PA, MA, CE, PB, PE, AL, SE, BA, MG, ES, RJ, SP, PR, SC, RS \\
\hline Vriesea bituminosa Wawra & & & & & $\mathrm{x}$ & VU & CE, BA, MG, ES, RJ, SP \\
\hline Vriesea carinata Wawra & & & & & $\mathrm{x}$ & VU & BA, MG, ES, RJ, SP, PR, SC, RS \\
\hline Vriesea drepanocarpa (Baker) Mez & & & $\mathrm{x}$ & $\mathrm{x}$ & $\mathrm{x}$ & VU & BA, ES, RJ, SP, PR, SC \\
\hline Vriesea gigantea Gaudich. & & & $\mathrm{x}$ & $\mathrm{x}$ & $\mathrm{x}$ & VU & PE, AL, BA, MG, ES, RJ, SP, PR, SC, RS \\
\hline Vriesea gradata (Baker) Mez & & & & & $\mathrm{x}$ & NA & MG, ES, RJ, SP \\
\hline Vriesea jonghei (K. Koch) E. Morren & & $\mathrm{x}$ & & $\mathrm{x}$ & & NA & BA, MG, RJ, SP, PR, SC \\
\hline Vriesea longiscapa Ule & & & & $\mathrm{x}$ & & NA & ES, RJ, SP \\
\hline Vriesea lubbersii (Baker) E. Morren & & & $\mathrm{x}$ & $\mathrm{x}$ & $\mathrm{x}$ & NA & ES, MG, RJ, SP, SC \\
\hline Vriesea pauperrima E. Pereira & & & & & $\mathrm{x}$ & NA & BA, MG, ES, RJ, SP, PR, RS \\
\hline Vriesea philippocoburgii Wawra & & & & $\mathrm{x}$ & $\mathrm{x}$ & VU & RJ, SP, PR, SC, RS \\
\hline $\begin{array}{l}\text { Vriesea procera (Mart. ex Schult. \& } \\
\text { Schult.) f. Wittm. }\end{array}$ & $\mathrm{x}$ & $\mathrm{x}$ & $\mathrm{x}$ & $\mathrm{x}$ & $\mathrm{x}$ & VU & $\begin{array}{l}\text { PI, CE, RN, PB, PE, AL, SE, BA, MG, ES, RJ, SP, PR, SC, } \\
\text { RS }\end{array}$ \\
\hline
\end{tabular}


Tabela 1. Continuação...

\begin{tabular}{|c|c|c|c|c|c|c|c|}
\hline Espécies & $\mathbf{A}$ & B & $\mathbf{C}$ & D & $\mathbf{E}$ & CA & Ocorrência no Brasil \\
\hline Vriesea rodigasiana E. Morren & $\mathrm{x}$ & $\mathrm{x}$ & & $\mathrm{x}$ & $\mathrm{x}$ & VU & CE, PE, BA, ES, RJ, SP, SC, PR, RS \\
\hline Vriesea rubyae E. Pereira & & & & $\mathrm{x}$ & $\mathrm{x}$ & VU & $\mathrm{RJ}$ \\
\hline Vriesea scalaris E. Morren & & & & $\mathrm{x}$ & $\mathrm{x}$ & VU & PE, BA, MG, ES, RJ, SP, PR, SC \\
\hline Vriesea secundiflora Leme & & & & & $\mathrm{x}$ & NA & $\mathrm{RJ}, \mathrm{SP}$ \\
\hline Vriesea simplex (Vell.) Beer & & & $\mathrm{x}$ & $\mathrm{x}$ & & NA & BA, ES, RJ, SP \\
\hline Vriesea unilateralis (Baker) Mez & & & $\mathrm{x}$ & $\mathrm{x}$ & $\mathrm{x}$ & $\mathrm{EP}$ & ES, RJ, SP, PR, SC \\
\hline Vriesea vagans (L.B. Sm.) L.B. Sm. & & & $\mathrm{x}$ & $\mathrm{x}$ & $\mathrm{x}$ & NA & MG, ES, RJ, SP, PR, SC, RS \\
\hline \multicolumn{8}{|l|}{ Subfamília Bromelioideae } \\
\hline Bromeliaceae indeterminada & & & & & $\mathrm{x}$ & - & - \\
\hline Aechmea coelestis (K. Koch) E.Morren & & & & $\mathrm{x}$ & & NA & ES, RJ, SP, PR, SC \\
\hline Aechmea distichantha Lem. & $\mathrm{x}$ & $\mathrm{x}$ & & $\mathrm{x}$ & $\mathrm{x}$ & VU & MG, RJ, SP, PR, SC, RS \\
\hline Aechmea gracilis Lindm. & $\mathrm{x}$ & $\mathrm{x}$ & $\mathrm{x}$ & $\mathrm{x}$ & $\mathrm{x}$ & VU & RJ, SP, PR, SC \\
\hline Aechmea nudicaulis (L.) Griseb. & $\mathrm{x}$ & $\mathrm{x}$ & $\mathrm{x}$ & $\mathrm{x}$ & $\mathrm{x}$ & VU & PB, PE, MG, ES, RJ, SP, PR, SC, RS \\
\hline Aechmea organensis Wawra & $\mathrm{x}$ & & & $\mathrm{x}$ & & NA & MG, RJ, SP, PR \\
\hline Aechmea pectinata Baker & $\mathrm{x}$ & & & $\mathrm{x}$ & $\mathrm{x}$ & $\mathrm{R}$ & RJ, SP, PR, SC \\
\hline Aechmea weilbachii Didrich & & $\mathrm{x}$ & & $\mathrm{x}$ & $\mathrm{x}$ & NA & RJ, ES \\
\hline Ananas bracteatus (Lindl.) Schult. Schult. f. & & & $\mathrm{x}$ & $\mathrm{x}$ & $\mathrm{x}$ & NA & PE, AL, MG, ES, RJ, SP, PR, SC, RS \\
\hline Ananas comosus (L.) Merr. & & & & & $\mathrm{x}$ & NA & Exótica \\
\hline Billbergia amoena (Lodd.) Lindl. & $\mathrm{x}$ & $\mathrm{x}$ & & $\mathrm{x}$ & $\mathrm{x}$ & NA & BA, ES, MG, GO, RJ, SP, PR, SC \\
\hline Billbergia pyramidalis (Sims) Lindl. & & & $\mathrm{x}$ & $\mathrm{x}$ & $\mathrm{x}$ & NA & BA, MG, ES, RJ, SP \\
\hline Billbergia zebrina (Herb.) Lindl. & & & & $\mathrm{x}$ & $\mathrm{x}$ & VU & MG, RJ, SP, PR, SC, RS \\
\hline Bromelia anthiacantha Bertol. & $\mathrm{x}$ & $\mathrm{x}$ & & $\mathrm{x}$ & $\mathrm{x}$ & NA & BA, MG, ES, RJ, SP, PR, SC, RS \\
\hline $\begin{array}{l}\text { Canistropsis cff. billbergioides (Schult. } \\
\& \text { Schult. f.) Leme }\end{array}$ & & & & $\mathrm{x}$ & $\mathrm{x}$ & NA & BA, ES, RJ, SP, PR, SC \\
\hline $\begin{array}{l}\text { Canistropsis microps (E. Morren ex } \\
\text { Mez) Leme }\end{array}$ & $\mathrm{x}$ & $\mathrm{x}$ & $\mathrm{x}$ & $\mathrm{x}$ & $\mathrm{x}$ & $\mathrm{R}$ & RJ, SP \\
\hline $\begin{array}{l}\text { Edmundoa ambigua (Wand. \& Leme) } \\
\text { Leme }\end{array}$ & & & & $\mathrm{x}$ & $\mathrm{x}$ & NA & RJ, SP \\
\hline Edmundoa lindenii (Regel) Leme & $\mathrm{x}$ & & & $\mathrm{x}$ & $\mathrm{x}$ & VU & MG, ES, RJ, SP, PR, SC, RS \\
\hline $\begin{array}{l}\text { Neoregelia cruenta (R. Graham) } \\
\text { L.B.Sm. }\end{array}$ & $\mathrm{x}$ & & & $\mathrm{x}$ & & VU & $\mathrm{BA}, \mathrm{ES}, \mathrm{RJ}, \mathrm{SP}$ \\
\hline Neoregelia hoehneana L.B.Sm. & & & $\mathrm{x}$ & $\mathrm{x}$ & $\mathrm{x}$ & VU & RJ, SP \\
\hline Neoregelia johannis (Carrière) L.B.Sm. & & & $\mathrm{x}$ & $\mathrm{x}$ & $\mathrm{x}$ & NA & $\mathrm{RJ}, \mathrm{SP}$ \\
\hline Nidularium inocentii Lem. & $\mathrm{x}$ & $\mathrm{x}$ & & $\mathrm{x}$ & $\mathrm{x}$ & $\mathrm{EP}$ & BA, RJ, SP, PR, SC, RS \\
\hline Portea petropolitana (Wawra) Mez & $\mathrm{x}$ & $\mathrm{x}$ & & $\mathrm{x}$ & & NA & BA, ES, MG, RJ \\
\hline $\begin{array}{l}\text { Pseudananas sagenarius (Arruda } \\
\text { Camara) Camargo }\end{array}$ & & & & $\mathrm{x}$ & & NA & CE, PE, AL, BA, MS, MT, MG, ES, RJ, SP, PR, SC \\
\hline Quesnelia arvensis (Vell.) Mez & & $\mathrm{x}$ & & $\mathrm{x}$ & & NA & RJ, SP, PR \\
\hline $\begin{array}{l}\text { Quesnelia marmorata (Lem.) R.W. } \\
\text { Read }\end{array}$ & & & & $\mathrm{x}$ & & NA & ES, RJ, SP \\
\hline $\begin{array}{l}\text { Quesnelia quesneliana (Brongn.) } \\
\text { L.B.Sm. }\end{array}$ & $\mathrm{x}$ & & & $\mathrm{x}$ & & NA & ES, MG, RJ \\
\hline Wittrochia superba Lindm. & & & & $\mathrm{x}$ & $\mathrm{x}$ & $\mathrm{EP}$ & RJ, SP, PR, SC \\
\hline Espécies & A & $\mathrm{B}$ & $\mathrm{C}$ & $\mathrm{D}$ & $\mathrm{E}$ & & \\
\hline Riqueza & 19 & 16 & 19 & 46 & 47 & & \\
\hline
\end{tabular}

A - Araújo \& Oliveira (1988); B - Fontoura et al. (1991); C - Almeida et al. (1998); D - Rocha et al. (2000). E - Projeto Ecologia de Comunidades de Bromeliaceae da Ilha Grande; F - Dados do presente projeto. (*) Espécie coletada por T.C. Rocha-Pessôa e C.F.D. Rocha e ainda em processo de identificação; (**) Espécie coletada e ainda em processo de identificação por E. M. Leme; (***) Espécie sem identificação e em cultivo. CA = categorias de ameaça; $\mathrm{NA}=$ não ameaçada; $\mathrm{EP}=$ em perigo; $\mathrm{VU}$ = vulnerável; $\mathrm{R}=$ rara. 


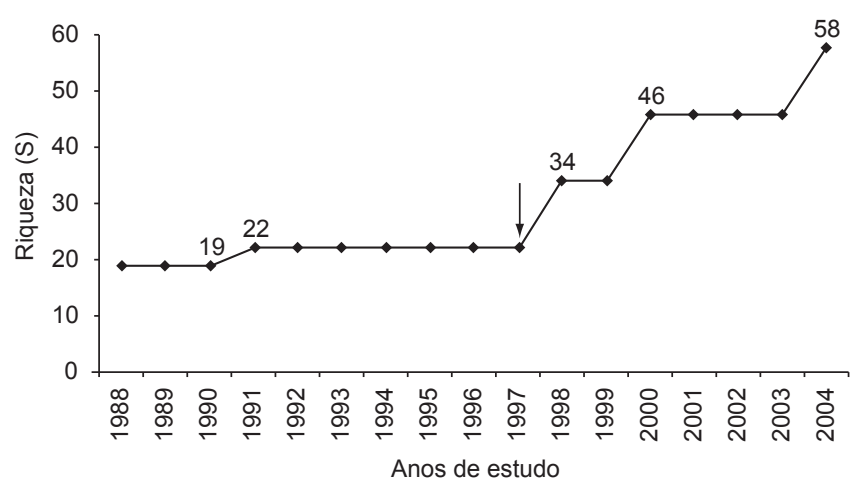

Figura 1. Incremento do conhecimento sobre a riqueza de espécies de Bromeliaceae na Ilha Grande, município de Angra dos Reis, RJ, ao longo do tempo de amostragem para registro de espécies. A seta indica o início dos estudos com metodologia de amostragem quantitativa na área.

Figure 1. Knowledge growth about the increase of Bromeliaceae species richness in Ilha Grande, municipality of Angra dos Reis, RJ, through out the sample period to register species. The arrow indicates the beginning of the use of quantitative sampling methods.

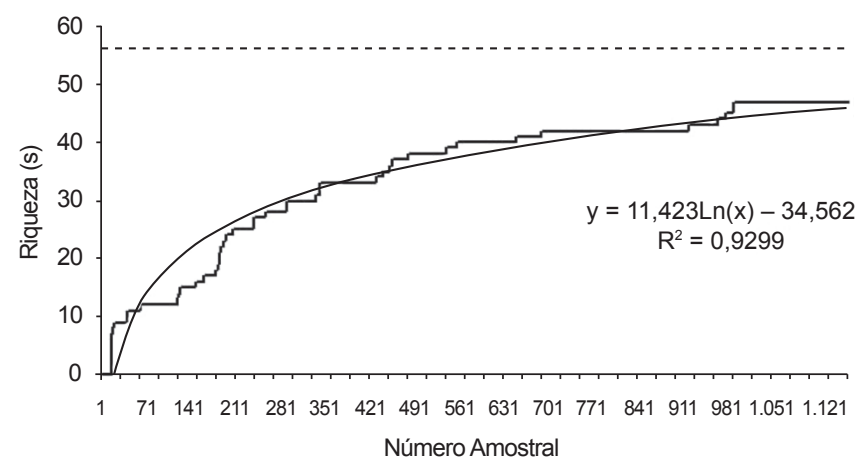

Figura 2. Aumento da riqueza de espécies de Bromeliaceae na Ilha Grande, município de Angra dos Reis, RJ, como função do número de parcelas amostradas na área ( $\mathrm{N}=1.170$ parcelas) e curva de ajuste segundo a função logarítmica que ajusta a reta. A fórmula descreve a função da curva ajustada. A linha pontilhada representa à riqueza total de espécies da família Bromeliaceae listadas para a Ilha Grande.

Figure 2. Increase of Bromeliaceae species richness in Ilha Grande, municipality of Angra dos Reis, RJ, as a function of the number of the sampling effort at the area ( $\mathrm{N}=1.170$ pieces) and the adjust curve according to the logarithmic function that adjusts the line. The formula describes the function of the adjusted curve. The dotted line represents the total species richness of Bromeliaceae family listed for Ilha Grande.

Com relação à ocorrência nas unidades da federação, a grande maioria ( $\mathrm{N}=48 ; 82,7 \%$ do total de espécies) ocorrem em quatro ou mais estados, indicando que apresentam uma distribuição que pode ser considerada ampla para o País. Segundo as listas oficiais, 28 (50,9\%) espécies listadas para a Ilha Grande foram categorizadas como não ameaçadas, $20(36,4 \%)$ como vulneráveis, cinco $(9,1 \%)$ como em perigo e dois $(3,6 \%)$ como raras.

\section{Discussão}

Os dados do presente estudo demonstraram que a Ilha Grande possui um elevado número de espécies de Bromeliaceae $(S=58)$, sendo que destas, $12(20,7 \%)$ são registros novos para a Ilha. As 58 espécies estão distribuídas em 16 gêneros, alguns deles endêmicos da Floresta Atlântica como, por exemplo, Wittrockia, Nidularium e
Canistropsis (Leme 1997). Dentre as subfamílias, Tillandsioideae e Bromelioideae são as que tiveram o maior número de espécies (29 e 28 espécies, respectivamente), o que era esperado, já que ambas são bem representadas na Floresta Atlântica (Leme 1997), diferente de Pitcairnioideae, que apresenta a maior parte das espécies ocorrendo na região amazônica (Benzing 2000). Os gêneros com maior número de espécies foram Vriesea e Tillandsia (18 e 11, respectivamente), considerados típicos da Floresta Atlântica, apesar de terem seu principal centro de origem nos Andes (Gentry 1982; Benzing 2000). Assim como estes gêneros, Aechmea também apresentou um elevado número de espécies ( $\mathrm{S}=7$ ), o que também era esperado, já que este gênero é descrito como típico da América tropical (Benzing 2000) e apresenta elevada riqueza de espécies na Floresta Atlântica, como acontece para o Estado do Rio de Janeiro como um todo (Fontoura et al. 1991).

O incremento de espécies à listagem de Bromeliaceae da Ilha Grande em função do tempo foi lento, mas relativamente constante desde o primeiro levantamento publicado para Ilha Grande. $\mathrm{O}$ aumento total foi de 38 espécies ao longo de aproximadamente 16 anos, equivalendo a $65,5 \%$ da listagem de espécies registradas. Este aumento pode estar relacionado especialmente a três fatores: à realização de estudos em diferentes áreas da Ilha Grande, à utilização de metodologias de amostragem quantitativas e ao tempo de estudo na área.

O estudo publicado por Araújo \& Oliveira (1988) foi realizado exclusivamente na área da RBEPS, que apesar de estar sendo estudada desde 1979, possui uma área relativamente pequena (3.600 ha) que corresponde a apenas 18,9\% da área total da Ilha Grande (Araújo \& Oliveira 1988), o número de espécies comparativamente baixo ( $\mathrm{S}=19$ ) encontrado neste estudo pode se dever a área restrita do mesmo. Isto também pode ser dito sobre o estudo de Almeida et al. (1998) $(\mathrm{S}=19)$ que se restringiu a área da Vila Dois Rios. No presente estudo foram realizadas amostragens, mesmo que pontuais, em todas as formações vegetais e em quase toda a área da Ilha Grande, o que constitui um dos principais fatores do aumento do número de espécies referidas.

Diferentes estudos têm demonstrado que a amostragem em diferentes pontos de uma localidade faz com que a curva espécie-área aumente mais rapidamente do que a amostragem intensiva em apenas uma área (Wilson et al. 1998, 1999, Phillips et al. 2003). Isso se deve especialmente ao fato de que, ao amostrar áreas distintas, amostra-se habitats com diferentes estruturas (Rosenzweig 1995), especialmente em termos de microclima (Huston 1999), solo (Franzmeier et al. 1969), altitude (Richardson et al. 2000) e abundância e diversidade da vegetação como um todo (McCarty et al. 1987). Essas diferenças estruturais entre áreas fazem com que haja uma mudança na composição de espécies entre elas (diversidade beta) (Whittaker 1972), já que diferentes espécies possuem respostas fisiológicas distintas às mudanças ambientais (Martin 1994; Scarano et al. 2001). O fato da amostragem ter sido realizada em diferentes áreas da Ilha Grande, incluindo uma grande variação na estrutura da vegetação, pode ter sido um dos principais fatores responsáveis pelo registro de espécies que ainda não haviam sido referidas e que ocupam porções específicas deste habitat insular.

Os diferentes métodos de amostragem de vegetação são utilizados (Queiroz 1998, Leis et al. 2003), em função do objetivo do estudo e da questão levantada. Cada método examina a paisagem ou a área em um diferente nível de resolução e extensão (Queiroz 1998, Leis et al. 2003), o que pode levar a interpretações distintas sobre o mesmo assunto (e.g. Magurran 1988, Queiroz 1998, Leis et al. 2003, Magnusson \& Mourão 2003). Além disso, diferentes métodos de amostragem podem levar à criação de curvas espécie-área com incrementos de espécies distintos, mesmo que o estudo seja realizado na 
mesma área e no mesmo período (Phillips et al. 2003). Dessa forma, é possível que o incremento na lista de espécies de Bromeliaceae registradas para a Ilha Grande possa ter sido afetado pelo tipo de amostragem realizada, já que a frequência do encontro de novas espécies é diretamente proporcional ao esforço amostral (Thompson et al. 2003). Os dois primeiros estudos que incluíram as espécies de Bromeliaceae da Ilha Grande utilizaram amostragem aleatória (Araújo e Oliveira 1988) ou dados de material depositado em herbários (Fontoura et al. 1991), que são métodos qualitativos utilizados para a elaboração de listas de espécies. Esses métodos têm papel fundamental como os primeiros indicadores da riqueza de uma área, mas apresentam como desvantagem a tendência da amostragem, já que há a possibilidade do coletor escolher localidades onde uma determinada espécie de interesse é encontrada ou onde a coleta é mais acessível (Margules et al. 2002), deixando de fora aquelas espécies raras ou que ocorrem em localidades de difícil acesso. Araújo (2000), analisando a flora das restingas do Rio de Janeiro também encontrou uma relação positiva entre o esforço de coleta e a riqueza de espécies. Em um outro estudo utilizando amostragem quantitativa, realizado em 13 restingas do leste brasileiro Cogliatti-Carvalho (2003) registrou cerca de $80 \%$ do total de espécies listadas para as localidades que amostrou, indicando que métodos quantitativos são eficazes para amostragens rápidas. Com a metodologia empregada no presente estudo, registramos um total de 45 espécies da família Bromeliaceae, o que equivale a 77,6\% do total das espécies listadas para a Ilha Grande, o que mostra que a utilização da metodologia quantitativa é satisfatória, já que, com ela, foi possível encontrar uma porcentagem elevada das espécies listadas. Assim, o uso de metodologias quantitativas que sejam realizadas em áreas pré-determinadas pode fazer com que a amostragem seja realizada da forma mais eficaz, permitindo um incremento de espécies mais acelerado, como demonstrado neste estudo.

Por outro lado, o fato da curva espécies-área não ter atingido um patamar indica que há a possibilidade do número total de espécies listadas ser alcançado ou ultrapassado, caso novas áreas não amostradas da Ilha Grande sejam vistoriadas com o mesmo método. Tackaberry et al. (1997) e Plotkin et al. (2000) sugeriram que a modelagem de curvas espécie-área pode não somente estimar a riqueza de uma área, mas também extrapolá-la, desde que a estrutura das áreas analisadas e das espécies seja levada em consideração.

Em termos de distribuição geográfica, a maior parte das espécies listadas apresenta ampla distribuição, ocorrendo em quatro ou mais unidades federativas. No entanto, os dados indicam que, possivelmente, o grande número de espécies listadas para a Ilha Grande possa ser uma representação em menor escala do que é observado para todo e leste brasileiro, onde são encontradas a maior parte das espécies de Bromeliaceae, especialmente entre os estados da Bahia e de São Paulo (Martinelli et al., 2008).

O número de espécies de Bromeliaceae listadas para a Ilha Grande e incluídas em alguma categoria de ameaça de extinção é coerente com o encontrado por Martinelli et al. (2008), que encontraram cerca de $40 \%$ das espécies da Floresta Atlântica. No entanto, como os próprios autores sugerem, esse valor pode estar sendo subestimado, já que ainda são poucas as informações sobre o estado das populações em ambientes naturais, o que muitas vezes dificulta a categorização e a conservação dessas espécies (Martinelli et al. 2008).

Os dados indicaram que a Ilha Grande apresenta uma elevada riqueza de espécies de Bromeliaceae, e que este resultado se deve não somente ao número de estudos e ao tempo decorrido desde os primeiros estudos incluindo o grupo na ilha, mas também à metodologia quantitativa utilizada no presente estudo. Por fim, os dados do presente estudo reforçam a idéia de que a Floresta Atlântica da Ilha Grande mantém uma significativa porção da biodiversidade de Bromeliaceae conhecida para o Estado do Rio de Janeiro.

\section{Agradecimentos}

Este estudo constitui parte dos resultados do Programa de Ecologia, Conservação e Manejo de Ecossistemas do Sudeste Brasileiro, Departamento de Ecologia, IBRAG, UERJ. E. Leme e A. Costa (Museu Nacional) identificaram as espécies de bromeliáceas estudadas. A M.C. Kiefer e R.D. Antonini pela leitura e sugestões sobre o manuscrito. Durante o estudo, A. F. N. Freitas recebeu bolsa de Doutorado (Registro CAPES No. 990207-7). C. F. D. Rocha recebeu bolsa de Produtividade e Auxílio à Pesquisa do Conselho Nacional do Desenvolvimento Científico e Tecnológico - CNPq (Processos № 307653/2003-0 e 477981/2003-8). Este estudo foi subvencionado com recursos da FAPERJ (processo $\mathrm{n}^{\circ}$ E-26/170.884/2002).

\section{Referências Bibliográficas}

ALHO, C.J.R., SCHNEIDER, M. \& VASCONCELLOS, L.A. 2002. Degree of threat to the biological diversity in the Ilha Grande State Park (RJ) and guidelines for conservation. Braz. J. Biol. 62(3):375-385.

ALMEIDA, D.R., COGLIATTI-CARVALHO, L. \& ROCHA, C.F.D. 1998. As bromélias da Mata Atlântica da Ilha Grande, RJ: composição e diversidade de espécies em três ambientes diferentes. Bromelia, 5(1-4):54-65.

ARAÚJO, D.S.D. 2000. Análise florística e fitogeográfica das restingas do Rio de Janeiro. Tese de Doutorado, Universidade Federal do Rio de Janeiro, Rio de Janeiro.

ARAÚJO, D.S.D. \& OLIVEIRA, R.R. 1988. Reserva Biológica da Praia do Sul (Ilha Grande, Estado do Rio de Janeiro): lista preliminar da Flora. Acta Bot. Bras. 1(2):83-94.

BENZING, D.H. 2000. Bromeliaceae: profile of an adaptative radiantion. Cambridge University Press, New York.

BITTENCOURT, E.B. \& ROCHA, C.F.D. 2002. Spatial use of rodents (rodentia: Mammalia) host body surface by ectoparasites. Braz. J. Biol. 62(3):419-425.

COGLIATTI-CARVALHO, L. 2003. Bromeliaceae em restingas da costa brasileira: variação interhábitat nos parâmetros da ecologia da taxocenose. Tese de doutorado, Universidade Federal do Rio de Janeiro, Rio de Janeiro.

FERREIRA Jr, O. 2004. GPS Track Maker.

FONTOURA, T., COSTA, A. \& WENDT, T. 1991. Preliminary checklist of the Bromeliaceae of Rio de Janeiro State, Brazil. Selbyana. 12:5-45.

FRANZMEIER, D.P., PEDERSON, E.J., LONGWELL, T.J., BYRNE, J.G. \& LOSCHE, C.K. 1969. Properties of some soils in the Cumberland Plateau as related to slope aspect and topography. Soil Sci. Soc. Am. Pro. 33:755-761.

GENTRY, A.H. 1982. Neotropical floristic diversity: phytogeographical connections between Central e South America, Pleistocene climate fluctuations or accident of the Andean orogeny? Ann. Mo Bot. Gard. 69(3):557-593.

HUSTON, M.A. 1999. Local processes and regional patterns: appropriate scales for understanding variation in the diversity of plants and animals. Oikos, 86(3):393-401.

Instituto Estadual de Meio Ambiente - IEMA. 2005. Decreto Estadual $\mathrm{n}^{\circ}$ 1499-R. Lista oficial das espécies da flora do estado do Espírito Santo ameaçadas de extinção. Diário Oficial do Estado, 14 de junho de 2005.

KLEIN, R.M. 1990. Espécies raras ou ameaçadas de extinção do estado de Santa Catarina. IBGE, Rio de Janeiro, p. 185-287.

LEIS, S.A., ENGLE, D.M., LESLIE Jr., D.M., FEHMI, J.S. \& KRETZER, J. 2003. Comparison of vegetation sampling procedures in a disturbed mixed-grass prairie. Proc. Okl. Acad. Sci. 83:7-15.

LEME, E. 1997. Canistrum: Bromélias da Mata Atlântica. Salamandra Consultoria Editorial Ltda, Rio de Janeiro.

MACIEL, N.C., ARAÚJO, D.S.D. \& MAGNANINI, A. 1984. Reserva Biológica Estadual da Praia do Sul (Ilha Grande, Angra dos Reis, 
RJ). Contribuição para o conhecimento da Fauna e Flora. Bol. FBCN, 19:126-148

MAGNUSSON, W.E. \& MOURÃO, G. 2003. Estatística sem matemática. Editora Planta, Londrina.

MAGURRAN, A.E. 1988. Ecological diversity and its measurement. University Press, Cambridge.

MARGULES, C.R., PRESSEY, R.L. \& WILLIAMS, P.H. 2002. Representing biodiversity: data and procedures for identifying priority areas for conservation. J. Biosciences. 27(4):309-326.

MARTIN, C.G. 1994. Physiological ecology of the Bromeliaceae. Bot. Rev. 60(1):1-35

MARTINELLI, G. 2006. Manejo de populações e comunidades vegetais: um estudo de caso na conservação de Bromeliaceae. In Biologia da conservação: essências (C.F.D. Rocha, H.G. Bergallo, M. Van Sluys \& M.A.S. Alves, orgs). Editora Rima, São Carlos, p. 479-503.

MARTINELLI, G., VIEIRA, C.M., GONZALEZ, M., LEITMAN, P., PIRATININGA, A., COSTA, A.F. \& FORZZA, R.C. Bromeliaceae da Mata Atlântica brasileira: lista de espécies, distribuição e conservação. Rodriguésia, 59(1):209-258.

McCARTY, B.C., HAMMER, C.A., KAUFFMAN, G.L. \& CANTINO, P.D. 1987. Vegetation patterns and structure of an old-growth forest in southeastern Ohio. B. Torrey Bot. Club. 114(1):33-45.

OLIVEIRA, R.R. 1999. Levantamento florístico das Matas de Encosta da Vila Dois Rios, Ilha Grande, RJ. Tese de Doutorado, Universidade Federal do Rio de Janeiro, Rio de Janeiro.

PHILLIPS, O. L., MARTÍNEZ, R.V., VARGAS, P. N., MONTEAGUDO, A.L., ZANS, M.E.C., SÁNCHEZ, W.G., CRUZ, A.P., TIMANÁ, M., YLIHALLA, M. \& ROSE, S. 2003. Efficient plot-based floristic assessment of tropical forest. J. Trop. Ecol. 19(6):629-645.

PLOTKIN, B.J., POTTS, M.D., LESLIE, N., MANOKARAN, N., LAFRANKIES, J. \& ASHTON, P.S. 2000. Species-area curves, apatial aggregation, and habitat specialization in tropical forest. J. Theor. Biol. 207(1):81-99.

POREMBSKI, S. \& BARTHLOTT, W. 1999. Pticairnia feliciana: the only indigenous african bromeliad. Harv. P. Bot. 4(1):175-183.

PRANCE, G.T., BEENTJE, H., DRANSFIELD, J. \& JOHNS, R. 2000. The tropical flora remains undercollected. Ann. Mo. Bot. Gard. 87(1):67-71.

QUEIROZ, W.T. 1998. Técnicas de amostragem em inventário florestal nos trópicos. FCAP; Serviço de Documentação e Informação, Belém.

RICHARDSON, B.A., RICHARDSON, M.J., SCATENA, F.N. \& McDOWELL, W.H. 2000. Effects of nutrient availiability and other elevational changes on bromeliad populations and their invertebrate communities in a humid tropical forest in Puerto Rico. J. Trop. Ecol. 16(2):167-188

ROCHA, C.F.D., COGLIATTI-CARVALHO, L., NUNES-FREITAS, A.F., ROCHA-PESSÔA, T.C. \& ALMEIDA, D.R. 2000. The bromeliad community of the Ilha Grande State Park, Rio de Janeiro State, Southeastern Brazil: species richness and ocurrence in na insular ecosystem. In Anais do V Simpósio de Ecossistemas Brasileiros (S. Watanabe, org). Publicações ACIESP, São Paulo, p. 126-132.
ROCHA, C.F.D., BERGALLO, H.G., ALVES, M.A.S. \& VAN SLUYS, M. 2003. A biodiversidade nos grandes remanescentes florestais do Estado do Rio de Janeiro e nas restingas da Mata Atlântica. RiMa Editora, São Carlos.

ROCHA-PESSÔA, T.C. \& ROCHA, C.F.D. 2008. Reproductive Cycle of Pitcairnia Flammea Lindl. (Bromeliaceae / Pitcairnioideae) in an Insular Atlantic Rainforest Area in Southeastern Brazil. Flora, 203: 229-233.

ROSENZWEIG, M.L. 1995. Species diversity in space and time. Cambridge University Press, Cambridge.

SCARANO, F.R., DUARTE, H.M., RIBEIRO, K.T., RODRIGUES, P.J.F.P. \& BARCELLOS, E.M.B. 2001. Four sites contrasting environmental stress in southeastern Brazil: realtions of species, life form diversity, and geographic distribution to ecophysiological parameters. Bot. J. Linn. Soc. 136(4):345-364

Secretaria Municial de Meio Ambiente - SEMA. 1995. Lista vermelha de plantas ameaçadas de extinção no estado do Paraná. SEMA/GTZ, Curitiba.

Secretaria Municial de Meio Ambiente - SEMA. 2002. Lista oficial das espécies plantas ameaçadas de extinção no estado do Rio Grande do Sul. Disponível em: <http://www.sema.rs.gov.br/sema/html/pdf/especiesameacadas $>$. (último acesso em 15/12/2008)

Secretaria Municial de Meio Ambiente - SEMA. 2004. Resolução SMA 48. Lista oficial das espécies da flora do estado de São Paulo ameaçadas de extinção. Diário Oficial do Estado de São Paulo. Disponível em: <http:// www.ibot.sp.gov.br/resolucao_sma48/resolucao48.htm>. (último acesso em 15/12/2008).

SMITH, L.B. 1955. The Bromeliaceae of Brazil. Smith. Miscelaneous Coll. 126:1-290.

TACKABERRY, R., BROKAW, N., KELLMAN, M. \& MALLORY, E. 1997. Estimating species richness in tropical forest: the missing species extrapolation technique. J. Trop. Ecol. 13:449-458.

THOMPSON, G.G., WITHERS, P.C., PIANKA, E.R. \& THOMPSON, S.A. 2003. Assessing biodiversity with species accumulation curves: inventories of small reptiles by pittrapping in Western Australia. Austral. Ecol. 28(4):361-383.

VELOSO, H.P., RANGEL-FILHO, A.L.R. \& LIMA, J.C.A. 1991 Classificação da vegetação brasileira, adaptada a um sistema universal. IBGE, Departamento de Recursos Naturais e Estudos Ambientais, Rio de Janeiro.

WHITTAKER, R. 1972. Evolution and measurement of species diversity. Taxon. 21:213-251.

WILSON, J.B., GITAY, H., STEEL, J.B. \& KING, W.M. 1998. Relative abundance distribution in plant communities: effects of species richness and of spatial scale. J. Veg. Sci. 9(2):213-220.

WILSON, J.B., STEEL, J.B., KING, W.M. \& GITAY, H. 1999. The effect of spatial scale on eveness. J. Veg. Sci. 10(4):463-468.

Recebido em 20/05/08 Versão Reformulada recebida em 23/03/09

Publicado em 17/04/09 